\title{
Síndrome pós Covid-19: estudo de caso
}

\author{
Post Covid-19 Syndrome: case study \\ Síndrome post Covid-19: estudio de caso
}

\author{
Veruska Cronemberger Nogueira Rebêlo \\ ORCID: https://orcid.org/0000-0002-5013-4432 \\ Universidade Estadual do Piauí, Brasil \\ E-mail: veruskanogueirarebelo@yahoo.com.br \\ Mikaelli Priscila Rosas Lemos \\ ORCID: https://orcid.org/0000-0001-6807-6085 \\ Universidade Estadual do Piauí, Brasil \\ E-mail: mikaellilemos@gmail.com \\ Eva Karoline Rodrigues da Silva \\ ORCID: https://orcid.org/0000-0001-8943-9180 \\ Universidade Estadual do Piauí, Brasil \\ E-mail: evakaroline56@gmail.com \\ Laiana Sepúlveda de Andrade Mesquita \\ ORCID: https://orcid.org/0000-0003-3256-7477 \\ Universidade Estadual do Piauí, Brasil \\ E-mail: 1aianaseulveda@hotmail.com \\ Patrícia Uchôa Leitão Cabral \\ ORCID: https://orcid.org/0000-0003-0702-6425 \\ Universidade Estadual do Piauí, Brasil \\ E-mail: patriciauchoa@ccs.uespi.br \\ Ana Flávia Machado de Carvalho \\ ORCID: https://orcid.org/0000-0002-6691-4804 \\ Universidade Estadual do Piauí, Brasil \\ Centro Universitário UniFacid Wyden, Brasil \\ E-mail: anaflaviaparaibana@hotmail.com \\ Rauirys Alencar de Oliveira \\ ORCID: https://orcid.org/0000-0001-5123-004X \\ Universidade Estadual do Piauí, Brasil \\ E-mail: rauirys@hotmail.com \\ Maura Cristina Porto Feitosa \\ ORCID: https://orcid.org/0000-0001-7095-7228 \\ Universidade Estadual do Piauí, Brasil \\ E-mail: mauraportofisio@hotmail.com \\ Nayana Pinheiro Machado de Freitas Coelho \\ ORCID: https://orcid.org/0000-0002-0134-0459 \\ Universidade Estadual do Piauí, Brasil \\ E-mail: nayanamachado@oi.com.br \\ Emilia Angela Lo Schiavo Arisawa \\ ORCID: https://orcid.org/0000-0003-3526-3890 \\ Universidade Vale do Paraíba, Brasil \\ Universidade Estadual do Piauí, Brasil \\ E-mail: mirela@univap.br
}

\section{Resumo}

A Síndrome Pós-Covid-19 (SPC) ou Covid longa (CL) é uma condição clínica oriunda da infecção pelo SARS-CoV-2 que algumas pessoas experimentam após o término da Covid- 19, com variedade de sintomas como fadiga, dor crônica, falta de ar, disfunção cognitiva, perda do olfato, tonturas, dores de cabeça e transtorno de ansiedade e estresse pós-traumático, impactando na qualidade de vida. Pacientes com doenças inflamatórias e autoimunes como Fibromialgia (FM) representam um grupo particularmente vulnerável ao desenvolvimento da CL devido alteração do Sistema Nervoso Autônomo (SNA), uso de glucocorticoides e do isolamento e sedentarismo impostos pela pandemia intensificarem dores crônicas, alterações emocionais e do sono. O objetivo desse estudo foi relatar um caso de SPC em paciente portador de FM com protocolo de tratamento composto por acupuntura e liberação miofascial no ambulatório de um hospital público em Teresina-PI. Os dados foram coletados com os instrumentos Depression Anxiety Stress (DASS-21), Mini Exame do Estado Mental (MEEM), Questionário Nórdico de Sintomas Osteomusculares (QNSO), Questionário de Avaliação de Qualidade de Vida (WHOQOL-BREF), Índice de Qualidade de Sono de Pittsburgh (PSQI), Escala Visual Analógica (EVA) e Algometria. Os resultados mostraram redução dos níveis de estresse, ansiedade e depressão, diminuição das dores musculares, aumento do LDP com redução do 
afastamento das atividades rotineiras, impactando na melhora da qualidade de vida geral e do sono. Conclui-se que o tratamento com acupuntura associada à liberação miofascial se apresenta como alternativa eficaz para melhoria da SPC em paciente portadora de FM.

Palavras-chave: Fibromialgia; Qualidade de vida; Saúde mental; Sistema Nervoso Autônomo; Covid-19.

\begin{abstract}
Post-Covid-19 Syndrome (PCS) or long Covid (CL) is a clinical condition arising from the SARS-CoV-2 infection that some people experience after the end of Covid-19, with a variety of symptoms such as fatigue, chronic pain, shortness of breath, cognitive dysfunction, loss of smell, dizziness, headaches and anxiety disorder and post-traumatic stress, impacting quality of life. Patients with inflammatory and autoimmune diseases such as Fibromyalgia (FM) represent a particularly vulnerable group to the development of LC due changes in the Autonomic Nervous System (ANS), use of glucocorticoids and the isolation and sedentary lifestyle imposed by the pandemic intensify chronic pain, emotional and sleep changes. The aim of this study was to report a case of PCS in a patient with FM to which a treatment protocol consisting of acupuncture and myofascial release at the outpatient clinic of a public hospital in Teresina-PI. Data were collected using Depression Anxiety Stress (DASS-21), Mini Mental State Examination (MMSE), Nordic Musculoskeletal Symptom Questionnaire (NSO), Quality of Life Assessment Questionnaire (WHOQOL-BREF), Quality Index Sleep Test (PSQI), Visual Analog Scale (VAS) and Algometry. The results showed a reduction in stress, anxiety and depression levels, a decrease in muscle pain, an increase in PPT with a reduction in withdrawal from routine activities, impacting on the improvement of the general quality of life and sleep. It is concluded that acupuncture treatment associated with myofascial release is an effective alternative for improving PCS in patients with FM.
\end{abstract}

Keywords: Fibromyalgia; Quality of life; Mental health; Autonomic Nervous System; Covid-19.

\title{
Resumen
}

El Síndrome Post Covid-19 (PCS) o Covid largo (CL) es una condición clínica derivada de la infección por SARSCoV-2 que experimentan algunas personas después del fin del Covid-19, con una variedad de síntomas como fatiga, dolor crónico, dificultad para respirar, disfunción cognitiva, pérdida del olfato, mareos, dolores de cabeza y trastorno de ansiedad y estrés postraumático, impactando la calidad de vida. Los pacientes con enfermedades inflamatorias y autoinmunes como la Fibromialgia (FM) representan un grupo particularmente vulnerable al desarrollo de CL debido a los cambios en el Sistema Nervioso Autónomo (SNA), el uso de glucocorticoides y el aislamiento y sedentarismo impuestos por la pandemia intensifican el dolor crónico, cambios emocionales y del sueño. El objetivo de este estudio fue relatar un caso de SCP en un paciente con FM com un protocolo de tratamiento que consistente en acupuntura y liberación miofascial en el ambulatorio de un hospital público de Teresina-PI. Los datos se recopilaron mediante depresión, ansiedad, estrés (DASS-21), miniexamen del estado mental (MMSE), cuestionario nórdico de síntomas musculoesqueléticos (NSO), cuestionario de evaluación de la calidad de vida (WHOQOL-BREF), prueba del índice de calidad del sueño (PSQI), Visual Analog Escala (EVA) y Algometría. Los resultados mostraron una reducción en los niveles de estrés, ansiedad y depresión, disminución del dolor muscular, aumento del PPT con reducción del retiro de las actividades rutinarias, impactando en la mejora de la calidad de vida general y del sueño. Se concluye que el tratamiento de acupuntura asociado a liberación miofascial es una alternativa eficaz para mejorar el PCS en pacientes con FM.

Palabras clave: Fibromialgia; Calidad de vida; Salud mental; Sistema Nervioso Autónom; Covid-19.

\section{Introdução}

Síndrome Pós-Covid-19 (SPC) ou Covid longa (CL) consiste na persistência dos sintomas por semanas ou meses após a infecção aguda pelo SARS-CoV-2, considerada como condição inflamatória difusa e multissistêmica, pode estar associada a alterações no Sistema Nervoso Central e musculoesquelético (Chen, 2020; Karsten et al., 2020). Além do período de incubação da doença e manifestação dos sinais clínicos, SPC acarreta ao indivíduo maior sofrimento pelo aparecimento de sequelas que retardam esse quadro, causando infecção multissistêmica pelo acometimento de tecidos vitais como o cardíaco, muscular e nervoso, incluindo alterações psicológicas (Gerônimo et al., 2021). As evidências clínicas e científicas sobre os efeitos subagudos e a longo prazo da SPC estão evoluindo, com relatos de sintomas multivariadas que incluem dor crônica, fraqueza muscular, dificuldade para respirar e déficits cognitivos (alterações de memória e fadiga mental), fadiga intensa, transtornos de ansiedade e estresse pós-traumático e sintomas neurológicos (perda do olfato, tonturas e dores de cabeça) que podem persistir por semanas ou meses, impactando diretamente na qualidade de vida (Velavan \& Meyer, 2020). As estratégias de manejo são variadas de acordo com o perfil sintomático de cada paciente com o objetivo de atenuar o risco de complicações (Silveira et al., 
2021). Pacientes acometidos por doenças inflamatórias e autoimunes, como a Fibromialgia (FM), representam um grupo vulnerável à CL devido a intensificação da sintomatologia, pelo uso de glucocorticoides na terapia imunossupressora, além da disautonomia causada pelo aumento do tônus simpático e a diminuição do estímulo parassimpático. O isolamento social e o sedentarismo impostos pela pandemia também são considerados fatores contribuintes para a síndrome por intensificar, a fadiga pós-Covid, dores crônicas musculoesqueléticas e alterações emocionais e do sono (Misra et al., 2020; Silva \& Sousa, 2020).

A FM é uma síndrome idiopática caracterizada por quadro crônico de dor musculoesquelética e sensibilidade generalizada, além de fadiga, distúrbios do sono, rigidez matinal, parestesias de extremidades, sensação subjetiva de edema e distúrbios cognitivos, de difícil tratamento, com importante prevalência na população geral (Wolfe et al., 2010). A qualidade de vida dos pacientes em idade produtiva de trabalho está diminuída, pois, além da dor, fadiga e fraqueza subjetiva levam a incapacidade para o trabalho. Na patogênese dessa síndrome múltiplos fatores e mecanismos podem estar envolvidos, e pesquisas demonstram que a disautonomia pode ter um importante papel na fisiopatologia da FM, embora não esteja claro se é causa ou efeito ou um componente deste mosaico. As alterações do SNA podem orientar uma abordagem terapêutica que busque equilibrar o sistema simpático alterado (Adler \& Geenen, 2005). A dor frequentemente não é aliviada com o tratamento farmacológico e os pacientes com FM optam por práticas integrativas como a acupuntura e liberação miofascial, em razão da boa tolerância e baixo risco de efeitos adversos (de Melo et al., 2013; Marchesini et al., 2014; Dias et al., 2016).

A acupuntura consiste na inserção de agulhas em pontos anatômicos específicos do corpo, com o objetivo de produzir efeito terapêutico (Liu et al., 2020). O contato com a acupuntura incentiva uma postura ativa e a visão crítica dos pacientes sobre seu próprio corpo, propiciando melhor autocuidado (Rizzi Cintra, \& Figueiredo, 2010). Diversos estudos apontam que a acupuntura reduz custos e consumo de medicamentos alopáticos (Vas et al., 2002). Nesse sentido, a acupuntura entra como opção de tratamento, pelo estímulo de pontos energéticos espalhados pelo corpo, ao longo dos meridianos, que visa à promoção, à manutenção e à recuperação da saúde, podendo ser utilizada de forma única ou integrada com outros recursos terapêuticos (Ferreira, 2020).

A Liberação Miofascial (LMF) é uma técnica manual realizada por profissionais de saúde, de característica passiva, que utiliza as mãos, cotovelos, dedo ou instrumentos específicos, restaurando uma disfunção somática com o intuito de reorganizar a fáscia e para atingir a homeostase naquela região (Da Silva et al., 2017; Barreto et al., 2019).

Tendo em vista que os pacientes acometidos pela SPC expressam necessidades específicas de reabilitação, que merecem uma abordagem sistemática baseadas em evidências científicas (Ceravolo et al., 2020), o objetivo desse estudo foi relatar um caso de SPC em paciente portador de FM com protocolo de tratamento composto por acupuntura e liberação miofascial, visando comprovar seu potencial como método terapêutico, não farmacológico e menos invasivo, focado na promoção da saúde integral do indivíduo.

\section{Metodologia}

Este relato de caso clínico de SPC é intervencional, de caráter qualiquantitativo, com amostra intencional, sendo orientado e realizado a partir das diretrizes tradicionais de estratégia de pesquisa. O presente estudo foi desenvolvido no ambulatório de um hospital público de referência no atendimento a pacientes com SPC em Teresina-PI. A pesquisa foi conduzida de acordo com os princípios éticos e orientações com base na Resolução nº 466/12 do Conselho Nacional de Saúde - CNS, após aprovado pelo Comitê de Ética em Pesquisa do Hospital Universitário da Universidade Federal do Piauí HUUFPI (CAAE: 42218620.6.0000.8050). O paciente recebeu todas as informações sobre os procedimentos que seriam realizados pelo Termo de Consentimento Livre e Esclarecido (TCLE), tendo seu início após e a assinatura desse documento. 
Inicialmente foi realizada criteriosa avaliação com coleta dos dados pessoais, da história relativa à síndrome Pós-Covid19 e exame físico, em ficha de avaliação específica, com campos para inserção dos dados coletados com a utilização dos seguintes instrumentos de avaliação:

Depression Anxiety Stress (DASS- 21): Questionário que avalia os estados emocionais de depressão, ansiedade e estresse, dividido em três escalas do tipo Likert, de 4 pontos, sendo que cada uma delas é constituída por 7 itens, tendo um total 21 itens. O resultado de cada escala é determinado pela soma dos resultados dos sete itens. A escala apresenta três notas, sendo uma por subescala, onde o mínimo é "0" e o máximo "21" (Martins et al., 2019; Formiga et al., 2021). Escala Visual Analógica (EVA): Escala de identificação da intensidade da dor, de fácil utilização e resultados reprodutíveis. Para garantir a fidedignidade na metodologia de aplicação da EVA, foi apresentada uma régua vertical de $10 \mathrm{~cm}$ que tem como extremos, expressões faciais que representam desde a "ausência de dor (0) até a dor insuportável (10)" (Lima et al., 2016; Pegoraro et al., 2019). Algometria: Técnica de mensuração fisiológica do sistema nociceptivo projetado para quantificar e documentar níveis de sensibilidade à palpação nos músculos, por meio da quantificação do Limiar de Percepção Dolorosa (LDP), considerado como um método conveniente para o estudo da integridade nociceptora em indivíduos normais ou portadores de síndromes álgicas. Nesse estudo, para avaliar o LDP utilizou-se o algômetro de pressão digital (Wagner Instuments®), com capacidade de dez quilogramas-força (kgf) e deformação de compressão de 10 cm (Lima et al., 2016; Prestes et al., 2020). Mini Exame do Estado Mental (MEEM): Breve teste de rastreio cognitivo para identificação de demência, constituído de duas partes, uma que abrange orientação, memória e atenção, com pontuação máxima de 21 pontos, e outra que aborda habilidades específicas como nomear e compreender, com pontuação máxima de 9 pontos, totalizando 30 pontos de escore. Valores mais altos de escore indicam maior desempenho cognitivo (de Melo, Barbosa \& Neri, 2017). Questionário Nórdico de Sintomas Osteomusculares (QNSO): Questionário que avalia os sintomas osteomusculares em pescoço, ombro, cotovelo, antebraço, punho/mão/dedos, região dorsal, região lombar, quadril/coxa, joelho e tornozelo/pé. Consiste em escolhas múltiplas ou binárias quanto a ocorrência de sintomas nas diversas regiões anatômicas mais comuns. O paciente deve relatar a ocorrência dos sintomas considerando os 12 meses e os sete dias precedentes à entrevista, bem como relatar a ocorrência de afastamento das atividades rotineiras no último ano. $\mathrm{O}$ índice de severidade de sintomas, para cada região anatômica, varia entre 0 e 4 , sendo que 0 representa ausência de sintomas. $\mathrm{O}$ índice 1 é atribuído para sintomas nos 12 meses precedentes ou nos sete dias precedentes; índice 2, para relatos de sintomas nos 12 meses e nos sete dias precedentes; índice 3, quando houve relato de sintomas nos sete dias ou nos 12 meses precedentes e afastamento das atividades; e o índice 4, para os registros de sintomas nos 12 meses e nos sete dias precedentes e afastamento das atividades (Braga et al., 2020; Lima et al., 2020). Questionário de Avaliação de Qualidade de Vida (WHOQOL-BREF): Trata-se de uma versão abreviada do WHOQOL-100 desenvolvido e recomendado pela OMS para avaliar a qualidade de vida em diversos grupos e situações. O instrumento é constituído por 26 questões, a primeira refere-se à qualidade de vida de modo geral e a segunda à satisfação com a própria saúde. As respostas seguem a escala de Likert (1 a 5), sendo que quanto maior a pontuação melhor a qualidade de vida. Além das duas questões iniciais, o questionário possui 24 facetas, as quais compõem 4 domínios que são: físico, psicológico, relações sociais e meio ambiente (Kluthcovsky \& Kluthcovsky, 2009; Teixeira et al., 2021). Índice de Qualidade de Sono de Pittsburgh (PSQI): Esse índice avalia a qualidade do sono durante o último mês e tem como objetivo fornecer uma medida de qualidade de sono padronizada que diferencia os pacientes entre "bons dormidores" e "maus dormidores". O questionário consiste em 19 questões agrupadas em 7 domínios (qualidade subjetiva do sono, a latência para o sono, a duração do sono, a eficiência habitual do sono, os transtornos do sono, o uso de medicamentos para dormir e a disfunção diurna), com pesos distribuídos numa escala de 0 a 3 . As pontuações destes componentes são somadas para produzirem um escore global, que varia de 0 a 21, onde, quanto maior a pontuação, pior a qualidade de sono, de forma que pontuações de 0 a 4 indicam boa qualidade de sono, de 5 a 10 indicam qualidade ruim e acima de 10, indicam distúrbio do sono (da Luz Dutra et al., 2021). 
O protocolo de tratamento foi composto por 12 atendimentos de acupuntura e liberação miofascial, 3 vezes na semana e 50 minutos de duração, atendendo a disponibilidade dos voluntários. Por sua vez, os pontos de acupuntura foram localizados anatomicamente de acordo com a descrição da Medicina Tradicional Chinesa (MTC), com o objetivo do de restabelecer o equilíbrio energético da paciente. $\mathrm{Na}$ intervenção com acupuntura foram selecionados 10 pontos de aplicação (VG20, E36, IG4, C7, P9, P7, F3, R6, VB40 e BP4), considerados pontos sistêmicos por apresentar indicações relacionadas à SPC: VG20relacionado ao lado espiritual e às emoções, incluindo ansiedade, funções cerebrais, distúrbios do sono e dor de cabeça; E36identificado por suas ações na homeostasia corporal, imunidade, e por atuar na psique; IG4- indicado para redução de dores, febre, alteração do SNC, estresse e distúrbios do sono; C7- relacionado com a parte emocional, incluindo depressão e insônia; P9- atua na respiração, dorsalgia, fraqueza geral e transtornos mentais; P7- dor e/ou fraqueza, tensão lombalgia, anosmia e diversos aspectos psicológicos; F3- cefaleia, distúrbios do humor, dormência e dor nas extremidades inferiores; R6- regulação de transtornos hormonais, distúrbios do sono, enxaqueca e sudorese noturna; VB40- dor torácica, vômitos e atrofia muscular dos membros inferiores; BP4- dor e plenitude epigástrica, miocardite, menstruação irregular, convulsão e alterações psiquiátricas.

A aplicação do protocolo terapêutico seguiu os seguintes passos: os recipientes contendo as agulhas filiformes, esterilizadas ( 25 X $30 \mathrm{~mm}$, Dong Bang), foram deslacrados na presença da paciente. Após antissepsia do local selecionado (álcool 70\%), as agulhas foram inseridas com ajuda do mandril, perpendicularmente à pele. As agulhas permaneceram aplicadas por 20 minutos, obedecendo ao padrão de duração das sessões de acupuntura, sendo feita estimulação manual inicial (rotação e pistonagem) no sentido anti-horário até atingir o DeQui, (sensação de ardência, peso, dormência, parestesia leve ou dolorimento leve), que corresponde ao fenômeno da sensação do ponto de acupuntura ativado. Ao término do tempo estabelecido, foi realizada outra estimulação com a retirada da agulha. A paciente permaneceu em decúbito dorsal durante todo o período da intervenção e as agulhas foram inseridas bilateralmente em todos os pontos, com exceção do ponto $\mathrm{VG}_{20}$, onde a aplicação foi unilateral.

A LMF foi realizada com a paciente inicialmente em decúbito dorsal, para liberação sequencial das fáscias do músculo occipitofrontal, suboccipitais, escaleno, esternocleidomastoideo (ECOM), escapulo-torácico, peitoral maior e menor e diafragma. Foi realizada força de tração no sentido cranial por 20 segundos, repetindo a manobra três vezes. Posteriormente, a paciente foi posicionada em decúbito lateral para liberar o músculo grande dorsal, finalizando com o decúbito ventral para liberação dos músculos paravertebrais cervicais e trapézio. Cada atendimento de LMF teve 30 minutos de duração.

Salienta-se que os atendimentos foram realizados pela pesquisadora principal, fisioterapeuta acupunturista com experiência em MTC e especialização em traumato-ortopedia com ênfase em terapia manual. Após o término dos 12 atendimentos foi realizada reavaliação para avaliar a evolução do tratamento.

\section{Caso clínico}

A paciente L.N.P.B., 49 anos, sexo feminino, enfermeira, divorciada, portadora da síndrome de FM. A voluntária descreveu suas condições iniciais de dor devido à FM e aos sintomas que persistiram após infecção por Covid-19. Na avaliação inicial, realizada dia 21/11/2021, a paciente relatou que ao adquirir a Covid- 19 realizou tratamento domiciliar não sendo necessário internação. Apresentou $25 \%$ de comprometimento do pulmão, além de tosse, coriza e febre alta. Após a infecção, desenvolveu quadro hipertensivo, além de apresentar dor crônica, fadiga crônica, fraqueza muscular, déficits cognitivos como esquecimento e perda da concentração, alterações gastrointestinais, emocionais e do sono. Sua queixa principal no momento da avaliação foi insônia e lapsos de memória. Após o protocolo de intervenção composto por 12 sessões de atendimento com acupuntura e liberação miofascial a paciente relatou que o sono havia melhorado e que as dores haviam reduzido tanto no 
estado de repouso quanto nas tarefas da vida diária. Também houve relato de melhora na função gastrointestinal cognitiva e emocional.

Na avaliação inicial, a paciente apresentava os seguintes resultados ao Questionário DASS 21, como ilustra a Figura 1 abaixo: depressão: normal - 2/42, estresse: extremamente severo - 36/42 e ansiedade: extremamente severo - 32/42

Figura 1 - Distribuição dos dados de acordo com a avaliação inicial do nível de depressão, estresse e ansiedade da paciente.

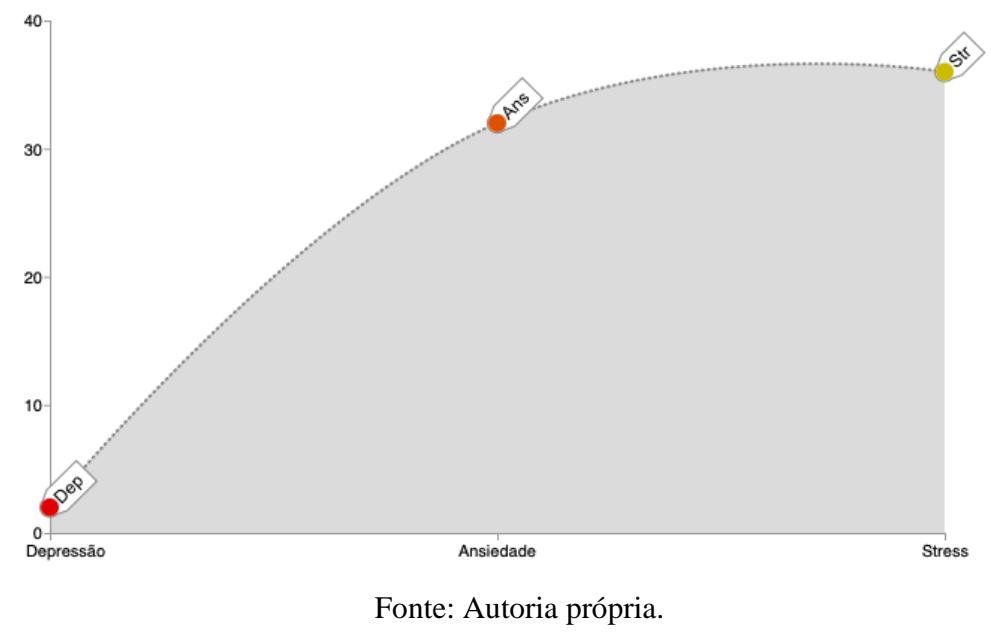

Na reavaliação realizada no dia 17/10/2021, após serem realizados os atendimentos de acordo com o protocolo de pesquisa, a paciente apresentou os seguintes resultados, como mostra a Figura 2: depressão: normal - 0/42, estresse: normal 6/42 e ansiedade: normal - 2/42.

Figura 2 - Distribuição dos dados de acordo com a reavaliação do nível de depressão, estresse e ansiedade da paciente.

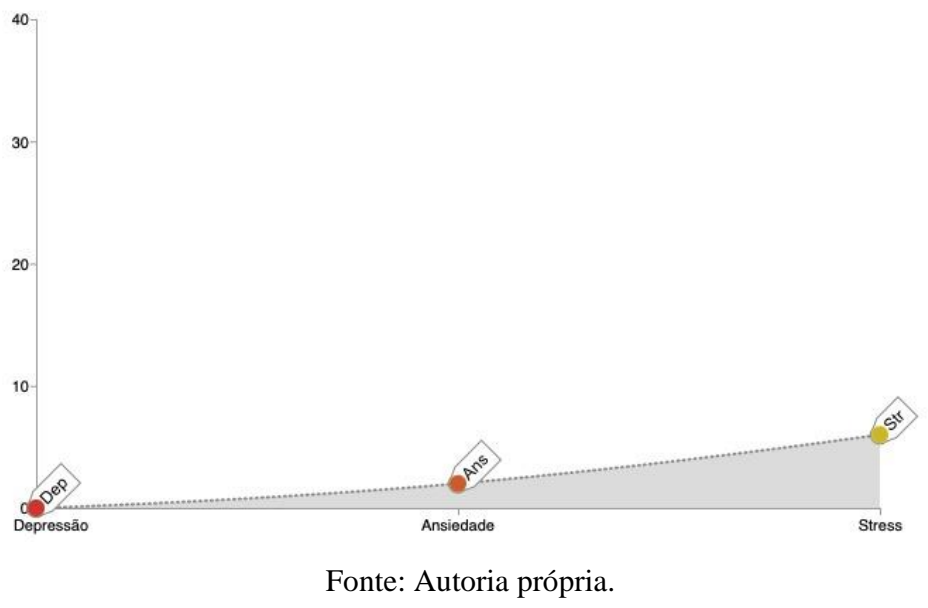

A Tabela 1 permite verificar que o relato de depressão se encontrava com nível normal antes do protocolo de intervenção, que foram reduzidos de 2/42 para 0/42, enquanto os níveis de ansiedade e estresse que estavam extremamente severos atingiram valores normais, com diminuição expressiva na pontuação de 32/42 para 2/42 e 36/42 para 6/42, respectivamente. 
Tabela 1 - Pontuação e classificação dos níveis de depressão, ansiedade e estresse por meio do DASS 21 na avaliação e reavaliação.

\begin{tabular}{lcc}
\hline \hline DASS 21 & Avaliação & Reavaliação \\
\hline Depressão & $2 / 42-$ Normal & $0 / 42-$ Normal \\
Ansiedade & $32 / 42-$ Extremamente severo & $2 / 42-$ Normal \\
Estresse & $36 / 42-$ Extremamente severo & $6 / 42-$ Normal \\
\hline \hline
\end{tabular}

Fonte: Autoria própria.

Quanto à intensidade da dor aferida com a EVA, a paciente referia dor grau 8 na avaliação. Na reavaliação a paciente apresentou melhora da condição clínica e diminuição da dor, referindo 5 como ilustra a Tabela 2.

Tabela 2 - Pontuação da intensidade da dor com EVA na avaliação e reavaliação.

\begin{tabular}{ccc}
\hline \hline EVA & Avaliação & Reavaliação \\
\hline 8 & 5 \\
\hline \hline
\end{tabular}

Fonte: Autoria própria.

Na algometria de pressão, observou-se importante melhora de dor, tensão e desconforto ao teste realizado. A Tabela 3 mostra o resultado da avaliação inicial e reavaliação, após o protocolo de intervenção, evidenciando melhora clínica do quadro e aumento do LDP no trapézio de 1.4 para $4.3 \mathrm{kgf}$, paravertebrais cervicais de 1.2 para $5.6 \mathrm{kgf}$ e ombros de 1.3 para $4.7 \mathrm{kgf}$.

Tabela 3 - Pontuação do LDP com algometria de pressão na avaliação e reavaliação.

\begin{tabular}{lcc}
\hline \hline LDP & Avaliação & Reavaliação \\
\hline Trapézio & $1.4 \mathrm{kgf}$ & $4.3 \mathrm{kgf}$ \\
Paravertebrais cervicais & $1.2 \mathrm{kgf}$ & $5.6 \mathrm{kgf}$ \\
Ombros & $1.3 \mathrm{kgf}$ & $4.7 \mathrm{kgf}$ \\
\hline \hline
\end{tabular}

Fonte: Autoria própria.

O MEEM foi utilizado com o intuito de avaliar a função cognitiva e rastreamento de quadros demenciais. Tanto na avaliação quanto na reavaliação, a paciente obteve pontuação máxima de 30/30 (Tabela 4).

Tabela 4 - Pontuação da função cognitiva com MEEM na avaliação e reavaliação.

\begin{tabular}{ccc}
\hline \hline MEEM & Avaliação & Reavaliação \\
\hline 30 & 30 \\
\hline \hline
\end{tabular}

Fonte: Autoria própria.

A avaliação das dores osteomusculares nas regiões do pescoço, ombros, punhos/dedos/mão, região dorsal, lombar, tornozelos e pés, utilizando o QNSO, observou-se que a paciente evoluiu do índice 4 (sintomas nos sete dias e nos 12 meses precedentes, além do afastamento das atividades) detectado na avaliação, para 2 (sintomas nos 12 meses e nos sete dias precedentes) na reavaliação, ilustrado na Tabela 5. 
Tabela 5 - Pontuação dos sintomas osteomusculares nas regiões anatômicas com QNSO na avaliação e reavaliações.

\begin{tabular}{|c|c|c|}
\hline QNSO & Avaliação & Reavaliação \\
\hline \multicolumn{3}{|c|}{ - Áreas com desconforto em 12 meses: } \\
\hline & Pescoço & Pescoço \\
\hline & Punhos/dedos/mão & Punhos/dedos/mão \\
\hline & Região dorsal & Região dorsal \\
\hline & Região lombar & Região lombar \\
\hline & Tornozelos e/ou pés & Tornozelos e/ou pés \\
\hline \multicolumn{3}{|c|}{ - Áreas com desconforto em 7 dias: } \\
\hline & Pescoço & Pescoço \\
\hline & Punhos/dedos/mão & Punhos/dedos/mão \\
\hline & Região dorsal & Região dorsal \\
\hline & Região lombar & Região lombar \\
\hline & Tornozelos e/ou pés & Tornozelos e/ou pés \\
\hline \multirow{2}{*}{\multicolumn{3}{|c|}{$\begin{array}{l}\text { - Atividades evitadas nos últimos } 12 \text { meses por } \\
\text { desconforto em: }\end{array}$}} \\
\hline & & \\
\hline \multicolumn{3}{|c|}{ Pescoço } \\
\hline \multicolumn{3}{|c|}{ Punhos/dedos/mão } \\
\hline \multicolumn{3}{|c|}{ Região dorsal } \\
\hline \multicolumn{3}{|c|}{ Região lombar } \\
\hline & Tornozelos e/ou pés & \\
\hline
\end{tabular}

Fonte: Autoria própria.

Ao se analisar os domínios do WHOQOL-BREF, observa-se que na reavaliação da paciente houve uma melhora na satisfação com a própria saúde, qualidade de vida geral, e nos domínios físicos e psicológicos, com modificações em suas classificações de ruim para boa. Na reavaliação do domínio das relações sociais, observa-se modificação de ruim para regular, no entanto não houve modificação com relação ao meio ambiente, mantendo-se assim como regular (Tabela 6).

Tabela 6 - Pontuação e classificação dos níveis da qualidade de vida geral e domínios com WHOQOL-BREF na avaliação e reavaliação.

\begin{tabular}{lcc}
\hline \hline WHOQOL-BREF (Domínios) & Avaliação & Reavaliação \\
\hline Qualidade de Vida Geral & 2 - Ruim & $4-$ Boa \\
Satisfação com sua saúde & 1 - Ruim & $4-$ Boa \\
Domínio Físico & 2 - Ruim & $4,1-$ Boa \\
Domínio Psicológico & 2,5 - Ruim & $4,2-$ Boa \\
Relações sociais & 2,4 - Ruim & $\mathbf{3}$ - Regular \\
Meio ambiente & 3,4 - Regular & $3,9-$ Regular \\
\hline \hline
\end{tabular}

Fonte: Autoria própria.

A paciente relatou melhora constante e gradativa da qualidade de sono, diminuição do tempo para adormecer e aumento das horas dormidas por noite após intervenção, na avaliação pelo PSQI. A pontuação global na avaliação indicava distúrbios do sono com média dos índices atingindo o grau 13. Na reavaliação, observou-se redução do valor para 2 , expressando boa qualidade do sono da paciente (Tabela 7). 
Tabela 7 - Pontuação da qualidade de sono com PSQI na avaliação e reavaliação.

\begin{tabular}{lcc}
\hline \hline PSQI & Avaliação & Reavaliação \\
\hline Qualidade do Sono & 3 & 1 \\
Latência do Sono & 3 & 0 \\
Duração do Sono & 3 & 0 \\
Eficiência Habitual do Sono & 1 & 0 \\
Distúrbios do Sono & 0 & 0 \\
Uso de medicação para dormir & 0 & 0 \\
Disfunção durante o dia & 1 & 3 \\
Pontuação Global do PSQI & 13 & 2 \\
Qualidade do Sono & Distúbio do sono & Boa qualidade do Sono \\
\hline \hline
\end{tabular}

Fonte: Autoria própria.

\section{Discussão}

A SPC, ou CL, é uma síndrome multissistêmica que pode se manifestar em pacientes recuperados da Covid-19 e impactar negativamente sua qualidade de vida (Silveira et al., 2021). Os resultados dos estudos conduzidos por Carvalho et al. (2021) e Mota et al. (2021) demonstraram que diversos aspectos da qualidade de vida dos indivíduos foram afetados após a infecção por Covid-19. Considera-se que o impacto é amplo e expressivo para as pessoas que se recuperam do vírus, especialmente porque tem relação com a presença de comorbidades, não sendo condição determinante ter desenvolvido a forma grave da doença, a CL independende da gravidade e da extensão da lesão resultante da infecção viral (Gerônimo et al. 2021).

Para Ladeia et al. (2020), o contexto pandêmico tem sido responsável por sintomas de ansiedade, depressão e estresse, resultando em diminuição da qualidade de vida e efeitos colaterais relacionados à saúde mental. Grupos de indivíduos susceptíveis são especialmente mais afetados, estando esse aspecto relacionado diretamente com o seu grau de envolvimento, vulnerabilidade ou perdas sociais que uma pandemia possa trazer, impactando negativamente o controle de comorbidades clínicas, em razão de incapacidades físicas, cognitivas e mentais associadas ao temor da doença. Garratt et al. (2021) destacaram o isolamento social imposto pela pandemia como fator de agravamento em pessoas que já se encontravam afetadas por doenças mentais.

Corroborando com esses achados, estudos apontaram que fatores associados ao gênero, profissão e estilo de vida podem piorar a qualidade de vida após o diagnóstico de infecção pelo SARS-CoV-2. Nesse contexto, as mulheres foram mais acometidas pela pandemia, com risco aumentado de ter baixa qualidade de vida, devido a maior pressão social nos cuidados familiares, por assumirem novas funções, gerando sobrecarga emocional e necessidade de tempos de reabilitação mais longos (Carvalho et al., 2021). Além do impacto na qualidade de vida pós-Covid, Guzmán-Muñoz et al., (2020) descreveram a presença de dor no corpo no gênero feminino.

Na pesquisa realizada por Silva et al., 2021, sobre repercussões da pandemia na qualidade de vida, os indivíduos com parceiros apresentaram maiores escores quando comparados aos solteiros. De acordo com Goldberg, Allen \& Smith (2021) a pandemia pelo Covid-19 causou estresse significativo em casais divorciados e separados com filhos, que enfrentaram tensões únicas em meio à pandemia com desafios legais, financeiros e de coparentalidade.

Nos estudos de Mota et al. (2021), os profissionais de saúde brasileiros tiveram qualidade de vida mais afetada durante a pandemia do que a prevalência observada em estudos internacionais na população em geral. Predominaram queixas relacionadas ao sono, uso de medicamentos para insônia, mudança na dieta alimentar, aumento do consumo de bebidas 
alcoólicas e mudança na prática de atividade física. Esses achados sugerem que profissionais de saúde expostos a fatores estressantes como a convivência com altas taxas de mortalidade, tiveram também piores condições de trabalho, relacionadas ao racionamento de equipamentos de proteção individual, falta de acesso a respiradores e outros suprimentos essenciais à saúde (Ladeia et al., 2020). Gavin et al. (2020) observaram altas taxas de sintomas de burnout em profissionais da saúde durante a pandemia da Covid-19, caracterizado por esgotamento físico e emocional, que se apresenta de variadas formas, como cansaço excessivo, dores de cabeça frequentes, dificuldade de concentração, depressão, ansiedade e uso indevido de substâncias.

Segundo Teixeira, de Freitas \& Carvalho (2020) os efeitos da dor crônica, pós-pandemia ocasionada pelo vírus Sars$\mathrm{CoV}-2$, na qualidade de vida dos pacientes são arrasadores. Estudo publicado no European Journal of Pain analisou pacientes com dor crônica e identificou que problemas como insônia, dificuldade de concentração, depressão e ansiedade. Pessoas com patologia reumática com dor crônica, tendem a manifestar emoções de revolta, raiva, ansiedade ou mesmo sintomas depressivos, que podem influenciar na sintomatologia negativa e na progressão e amplificação da doença.

Os sintomas de SPC incluem alteraçoes musculoesqueléticas como mialgia, dor articular e fadiga, bem como a presença de manifestações emocionais em indivíduos com diagnóstico de FM. Observou-se elevada vulnerabilidade de pessoas com patologias associadas à síndrome de sensibilidade central frente a infecção pelo vírus SARS-CoV-2 (Cercas-Lobo \& Deniel-Rosanas, 2021).

As dores crônicas e a FM apresentam uma característica fisiopatológica com o SNA, principalmente pelo aumento do tônus simpático e diminuição do estímulo parassimpático, gerando disautonomia. Quando o sistema simpático começa a ter predomínio, o SNA não terá mais flexibilidade, pois esse sistema autonômico desempenha importante papel na homeostasia do organismo. Com essa hipertonia simpática, devido a disfunção na membrana duramáter, as dores crônicas e a FM terão grandes chances de se desenvolverem (Favareto, 2019). Adler e Geenen (2005) referem que a hiperatividade simpática presente na FM indica aumento do impulso simpático ou alteração no controle normal da atividade do SNA pelo sono. Para Cohen et al. (2000) a hiperatividade simpática no repouso poderia estar parcialmente relacionada a sintomas como fadiga, distúrbios do sono, parestesias e síndrome do cólon irritável.

Para de Melo et al. (2020) a diversidade de técnicas empregadas para a FM, assim como a variabilidade em sua aplicação, impossibilita a avaliação padronizada, sugerindo a proposição de um protocolo de referência para essa situação clínica. Na pandemia pelo Covid-19, em regiões onde os métodos terapêuticos da MTC foram altamente utilizados, houve redução na gravidade dos casos e aumento da taxa de cura e alta hospitalar (Liu et al., 2020). Observou-se, em alguns estudos, resultados relevantes em pacientes tratados com acupuntura, com melhora no padrão do sono, disposição e estado emocional, resultando na diminuição do uso de fármacos (Ferreira, 2020). No estudo de revisão conduzido por de Almeida Oliveira, Campos \& Alves (2021) evidenciou-se o efeito da acupuntura na redução da dor e melhora da qualidade de vida em pacientes com FM, resultados semelhantes aos encontrados no ensaio clínico randômico de Takiguchi et al. (2008), que verificaram a eficácia da acupuntura na melhora da dor, sono e qualidade de vida de pacientes fibromiálgicos.

Resende et al. (2021) afirmaram que embora os mecanismos neurofisiológicos da acupuntura sejam complexos, e alguns ainda necessitem de esclarecimentos, essa técnica tem ação na neuromodulação da dor somática e visceral, assim como na modulação das funções viscerais e neuroendócrinas. A acupuntura potencializa os mecanismos fisiológicos de inibição da dor em nível local, segmentar, hetero-segmentar e central. Em nível local/periférico a acupuntura ativa mecanorrecetores nos tecidos cutâneos, fasciais e musculares e as evidências histológicas demonstraram que os pontos são zonas de densa inervação com receptores neurológicos periféricos ( $\mathrm{Li}$ et al., 2004). O estímulo mecânico da agulha promove ação pró-inflamatória local, com liberaçõ de citocinas, prostaglandinas e bradicinina, contribuindo também para o acelerar do processo de cicatrização. No tratamento de disfunções neuromusculares ocorre, localmente, desativação dos pontos gatilho e síndrome de dor miofascial 
gerando relaxamento muscular, por meio de mecanismo similar ao reflexo de alongamento (White, Cummings \& Filshie, 2018; Takakura et al., 2010).

A introdução de uma agulha de acupuntura em nível segmentar induz estimulação de fibras aferentes (fibras Ia, Ib, II e III no músculo) que competem no nível do corno dorsal da medula com a transmissão da informação nociceptiva veiculada pelas fibras C/IV aos centros de processamento corticais/superiores, modulando a dor, com duração limitada, denominada Teoria do Portão da Dor (Melzack e Wall, 1965; Silva et al., 2011). A ativação das fibras A delta/ III promove a ativação dos feixes inibitórios descendentes pela liberação de opioides endógenos, promovendo analgesia mais potente e duradoura (White, Cummings \& Filshie, 2018).

Menezes, Moreira \& Brandão (2010) esclarecem que o efeito central da acupuntura consiste na desativação das áreas envolvidas na manutenção da dor crônica (Matriz da Dor ou Pain Matrix) e nas implicações motoras da mesma, ao nível do sistema límbico (componente emocional da dor), córtex pré-frontal (componente cognitivo da dor) e córtex sensitivo (componente sensorial da dor), córtex motor e cerebelo (componente motor da dor) e hipotálamo (componente autonômico). O SNA pode ser influenciado por mecanismos moduladores somáticos e autônomos, ativados pela acupuntura, na recepção periférica, paraespinhal, segmentar e central (Lund \& Lundberg, 2016). A homeostasia do reflexo somato-visceral envolve os ramos simpáticos e parassimpáticos do SNA, tanto em nível segmentar quanto encefálico (Noguchi, 2010).

Diversos estudos abordam as técnicas manuais como importantes aliadas no tratamento da FM. Dentre as técnicas mais conhecidas estão pompagens, massagem terapêutica, liberação miofascial, osteopatia e quiropraxia. Na liberação da fáscia, a combinação de tração manual e alongamentos combate as aderências fasciais, melhorando os sintomas da FM (Liptan et al., 2013; Ziani et al., 2017). Segundo Gondim \& Almeida (2018) as manobras de terapia manual, especialmente nos pontosgatilho (PG - "Trigger Points”), geram resultados satisfatórios em pacientes com FM no alívio da dor crônica, melhora da fadiga e sono, bem como redução da ansiedade e depressão, promovendo melhor qualidade de vida. Nogueira (2008) afirma que diversos mecanismos neurofisiológicos estão envolvidos com os efeitos das técnicas de terapia manual, como hipoalgesia, tanto por mecanismos periféricos quanto centrais e inibição do espasmo muscular, por influência na excitabilidade do motoneurônio e melhora do controle motor e repercussões no SNA.

Apesar dos mecanismos neurofisiológicos na LMF ainda permanecerem incertos, Meseguer et al. (2006) sugerem que o efeito analgésico da técnica se deve à estimulação de fibras A delta. Em diversos estudos as técnicas de tratamento para PG demonstraram a eficácia na redução de dor (Lewis \& Flynn, 2001; Fryer \& Hodgson, 2005) e efeitos neurofisiológicos do alongamento muscular passivo (Guissard et al., 2001), que ocorrem preferencialmente com contração muscular, sendo que apenas o alongamento muscular extremo pode estimular esta via (fibras Ib), causando repercussão no reflexo $\mathrm{H}$.

De acordo com Nogueira (2008) muitos estudos sobre LMF evidenciaram achados de hipoalgesia e alterações no SNA, a inervação sensitiva provém de receptores musculares sensíveis ao estiramento e de receptores livres contidos na fáscia muscular, entre fibras musculares e tendões. Os estímulos destes receptores livres são transmitidos por axônios mielinizados A delta (grupo III) e amielinizados tipo C (grupo IV). Durante a sintomatologia dolorosa observa-se maior ativação do SNA simpático, portanto técnicas de terapia têm demonstrado eficácia no equilíbrio desse sistema, regulando algias, readequando o tônus muscular, melhorando a circulação tecidual e diminuindo a ansiedade, impactando na qualidade de vida dos pacientes (Salgado \& Pereira, 2012)

O manejo da SPC é considerando um desafio clínico devido à diversidade de sinais e sintomas envolvidos, bem como o acometimento de diferentes sistemas. Portanto não há diretrizes internacionais baseadas em evidências a serem seguidas (Garg et al., 2021). O acompanhamento de indivíduos com CL deve ser flexível, com base nos problemas clínicos de cada paciente (Nice, 2020). O ambiente em que vivemos influencia nossos pensamentos, sentimentos e emoções. O medo e situações estressantes vivenciados na pandemia de Covid-19 influenciaram o aumento da atividade do sistema simpático, 
afetando o sistema imunológico, gerando desautonomia do SNA. Reestabelecendo a flexibilidade autonômica, por meio de técnicas de estimulação parassimpática, muitos mecanismos homeostáticos retornam ao normal (Favareto, 2019).

Os dados coletados no presente relato de caso forneceram uma visão mais sensível e aprofundada do impacto na qualidade de vida dos indivíduos com SPC, portadores de FM, incentivando a proposição de protocolo individualizado e personalizado associando acupuntura e liberação miofascial para redução da disautonomia e reequilíbrio do SNA.

\section{Conclusão}

De acordo com os resultados obtidos no relato de caso de paciente portador de FM com SPC conclui-se que o protocolo de tratamento proposto com associação de acupuntura e liberação miofascial se apresenta como alternativa eficaz, promovendo redução dos níveis de estresse, ansiedade e depressão, diminuição das dores musculares, aumento do LDP, com redução do afastamento das atividades rotineiras, impactando na melhora da qualidade de vida geral e do sono.

Este estudo apresenta limitações, principalmente por ser um relato de caso clínico, embora o rigor científico tenha sido aplicado em todas as etapas de análise, interpretação e descrição dos resultados. Faz-se necessária a replicação das avaliações realizadas, para mensurar o impacto do protocolo de tratamento proposto sobre os sintomas da SPC, visto que este tema ainda é pouco estudado e os achados dessa pesquisa precisam ser investigados e confirmados em maiores estudos populacionais.

Reforça-se a necessidade da disseminação do conhecimento no tocante ao protocolo de tratamento com a associação de acupuntura e liberação miofascial, bem como o comprometimento por parte dos profissionais de saúde na identificação dos sintomas da SPC em portadores de FM, além da notificação dos casos para os órgãos de saúde pública, para melhor desfecho terapêutico.

\section{Agradecimentos}

Os autores agradecem o apoio da UESPI, na pessoa da Profa. Dra. Luana de Moura Monteiro, coordenadora do curso de Fisioterapia. Agradecemos ao apoio logístico do HPM-PI que tem uma politica de valorização da ciência, e seu Diretor Geral, Ten. Cel-PM Marcos Rogério de Souza, bem como à coordenadora do Centro de Ensino, Pesquisa e Extensão, Major Maria Aparecida Batista Silva. Agradecemos, também, os fisioterapeutas do HPM-PI pela receptividade durante a coleta de dados, destacando à disponibilidade da Dra. Arlene de Pádua Costa Pinheiro, coordenadora do Ambulatório e Setor Pós-COVID e, de forma especial, à generosidade da paciente voluntária que se prontificou participar desta pesquisa.

\section{Referências}

Adler, G. K., \& Geenen, R. (2005). Hypothalamic-pituitary-adrenal and autonomic nervous system functioning in fibromyalgia. Rheumatic Disease Clinics, 31(1), 187-202.

Barreto, E., Ramos, D., Silva, F., \& Petrini, A. C. (2019). Liberação miofascial aumenta a flexbilidade muscular em atletas. DêCiência em Foco, 3(1), 129139.

Braga, R. S., da Silva Lima, R., Bacil, L. F., de Macedo, A. C. B., \& Motter, A. A. (2020). Efeitos da cinesioterapia laboral nos sintomas osteomusculares crônicos de servidores universitários de um setor da Universidade Federal do Paraná. Revista Pesquisa em Fisioterapia, 10(2), 172-181.

Carvalho, M. C. T., de Jesus, B. M. B., de Castro, V. L., \& Trindade, L. M. D. (2021). O impacto na qualidade de vida nos indivíduos pós Covid-19: O que mudou? Research, Society and Development, 10(14), e219101421769-e219101421769.

Ceravolo, M. G., De Sire, A., Andrenelli, E., Negrini, F., \& Negrini, S. (2020). Systematic rapid" living" review on rehabilitation needs due to COVID-19: update to March 31st, 2020. European journal of physical and rehabilitation medicine, 56(3), 347-353.

Cercas-Lobo, S., \& Deniel-Rosanas, J. (2021). COVID-19 persistente y síndrome de sensibilidad central. Atención Primaria Práctica, $3(3)$, 100095.

Cohen, H., Neumann, L., Shore, M., Amir, M., Cassuto, Y., \& Buskila, D. (2000, February). Autonomic dysfunction in patients with fibromyalgia: application of power spectral analysis of heart rate variability. In Seminars in arthritis and rheumatism. 29(4), 217-227. 
Chen, J. (2020). Pathogenicity and transmissibility of 2019-nCoV - a quick overview and comparison with other emerging viruses. Microbes and infection, 22(2), 69-71.

da Luz Dutra, L., de Aquino, A. C. N., da Silva, E. L., \& Barros, L. N. (2021). Avaliação do Índice de Qualidade do Sono de Pittsburgh em estudantes de Medicina: Uma revisão integrativa da literatura. Research, Society and Development, 10(8), e52410817530-e52410817530.

Da Silva, D. L., Monteiro, E. R., Neto, V. G. C., \& da Silva Triani, F. (2017). Efeitos da liberação miofascial sobre a flexibilidade: uma revisão sistemática. Journal of Health Sciences, 19(3), 200-204.

de Almeida Oliveira, I., Campos, M. A., \& Alves, R. B. (2021). Eficácia da Acupuntura como técnica complementar para redução da dor e melhora da qualidade de vida de pacientes com Fibromialgia. Revista Brasileira de Práticas Integrativas e Complementares em Saúde, 1(1), 61-72.

de Melo Barbosa, A., Neto, G. A. M., do Vale, L. A., \& Pirett, V. M. Acupuntura no tratamento da fibromialgia: uma revisão de literatura. Revista Movimenta, 6(2), 2013.

de Melo, D. M., Barbosa, A. J. G., \& Neri, A. L. (2017). Miniexame do Estado Mental: evidências de validade baseadas na estrutura interna. Avaliação psicológica, 16(2), 161-168.

de Melo, G. A., de Lima Araújo, G., de Vasconcelos, A. M., \& Torro, N. (2020). Recursos terapêuticos para a fibromialgia: uma revisão sistemática. Revista Contexto \& Saúde, 20(38), 49-56.

Dias, P. A., Guimarães, A. B. B., de Oliveira Albuquerque, A., de Oliveira, K. L., Cavalcante, M. L. C., \& Guimarães, S. B. (2016). Short-term complementary and alternative medicine on quality of life in women with fibromyalgia. Journal of integrative medicine, 14(1), 29-35.

Favareto, R. M. (2019). Influência da manipulação osteopática craniana, sobre o sistema nervoso autônomo mensurado pela neurometria funcional em pacientes com fibromialgia. Revista Científica de Neurometria, 5.

Ferreira, A. A. D. M. (2020). Protocolo de acupuntura preventiva para estimular imunidade frente à COVID-19. InterAmerican Journal of Medicine and Health, 3, 1-19.

Formiga, N. S., Franco, J. B. M., Oliveira, H. C. C., Prochazka, G. L., Beserra, T. K. P., Valin, C. G. P., \& Nascimento, R. L. (2021). Invariância fatorial, sensibilidade e diferenças da medida de ansiedade, estresse e depressão (DASS-21) em trabalhadores brasileiros. Research, Society and Development, 10(7), e26910715572-e26910715572.

Fryer, G., \& Hodgson, L. (2005). The effect of manual pressure release on myofascial trigger points in the upper trapezius muscle. Journal of Bodywork and movement therapies, 9(4), 248-255.

Garg, M., Maralakunte, M., Garg, S., Dhooria, S., Sehgal, I., Bhalla, A. S., \& Sandhu, M. S. (2021). The Conundrum of 'Long-COVID-19': A Narrative Review. International Journal of General Medicine, 14, 2491.

Garratt, A. M., Ghanima, W., Einvik, G., \& Stavem, K. (2021). Quality of life after COVID-19 without hospitalisation: Good overall, but reduced in some dimensions. The Journal of infection.

Gavin, B., Hayden, J., Adamis, D., \& McNicholas, F. (2020). Caring for the psychological well-being of healthcare professionals in the Covid-19 pandemic crisis. Ir Med J, 113(4), 51.

Gerônimo, A. M. M., Comassetto, I., Andrade, C. R. A. G., \& da Silva, R. R. S. M. (2021). Além do SARS-CoV-2, as implicações da Síndrome Pós COVID19: o que estamos produzindo? Research, Society and Development, 10(15), e336101522738-e336101522738.

Goldberg, A. E., Allen, K. R., \& Smith, J. Z. (2021). Divorced and separated parents during the COVID-19 pandemic. Family process, 60(3), 866-887.

Gondim, S. S., \& Almeida, M. A. P. T. (2018). Os efeitos da massagem terapêutica manual em pacientes com a síndrome da fibromialgia. ID on line revista de psicologia, 12(39), 336-354.

Guissard, N., Duchateau, J., \& Hainaut, K. (2001). Mechanisms of decreased motoneurone excitation during passive muscle stretching. Experimental Brain Research, 137(2), 163-169.

Guzmán-Muñoz, E., Concha-Cisternas, Y., Oñate-Barahona, A., Lira-Cea, C., Cigarroa-Cuevas, I., Méndez-Rebolledo, G., \& Zapata-Lamana, R. (2020). Factores asociados a una baja calidad de vida en adultos chilenos durante la cuarentena por COVID-19. Revista médica de Chile, $148(12), 1759-1766$.

Jacomini, L. C. L., \& Silva, N. A. D. (2007). Disautonomia: um conceito emergente na síndrome da fibromialgia. Revista Brasileira de Reumatologia, 47, $354-361$.

Karsten, M., Matte, D. L., \& de Andrade, F. M. D. (2020). A pandemia da COVID-19 trouxe desafios e novas possibilidades para a Fisioterapia no Brasil: estamos preparados? Revista Pesquisa em Fisioterapia, 10(2), 142-145.

Kluthcovsky, A. C. G., \& Kluthcovsky, F. A. (2009). O WHOQOL-bref, um instrumento para avaliar qualidade de vida: uma revisão sistemática. Revista de Psiquiatria do Rio Grande do Sul, 31 .

Ladeia, D. N., da Silva, A. F., Gonçalves, B. B. S., Damasceno, C. M. C., Vieira, J. P. G., Silva, J. A. L., \& Lopes, A. G. (2020). Análise da saúde mental na população geral durante a pandemia de Covid-19. Revista Eletrônica Acervo Saúde, (46), e3925-e3925.

Lewis, C., \& Flynn, T. W. (2001). The use of strain-counterstrain in the treatment of patients with low back pain. Journal of Manual \& Manipulative Therapy, 9(2), 92-98.

Li, A. H., Zhang, J. M., \& Xie, Y. K. (2004). Human acupuncture points mapped in rats are associated with excitable muscle/skin-nerve complexes with enriched nerve endings. Brain research, 1012(1-2), 154-159. 
Lima, A. R. S., Portes, L. A., de Oliveira, N. C., \& Alfieri, F. M. (2016). Limiar de tolerância de dor à pressão, estilo de vida, força muscular e capacidade funcional em idosas com sarcopenia. CEP, 5858,001 .

Lima, T. B. W., Albuquerque, J. R., Fagundes, M. G., \& Coutinho, C. C. C. (2020). Prevalência de sintomas osteomusculares e qualidade de vida de trabalhadores técnicos administrativos. Rev. bras. med. trab, 45-50.

Liptan, G., Mist, S., Wright, C., Arzt, A., \& Jones, K. D. (2013). A pilot study of myofascial release therapy compared to Swedish massage in fibromyalgia. Journal of bodywork and movement therapies, 17(3), 365-370.

Liu, W. H., Guo, S. N., Fang, W. A. N. G., \& Yang, H. A. O. (2020). Understanding of guidance for acupuncture and moxibustion interventions on COVID-19 issued by CAAM. World journal of acupuncture-moxibustion, 30(1), 1-4.

Lund, I., \& Lundeberg, T. (2016). Mechanisms of acupuncture. Acupuncture and Related Therapies, 4 (4), 26-30.

Marchesini Stival, R. S., Cavalheiro, P. R., Stasiak, C., Galdino, D. T., Hoekstra, B. E., \& Schafranski, M. D. (2014). Acupuntura na fibromialgia: um estudo randomizado-controlado abordando a resposta imediata da dor. Revista Brasileira de Reumatologia, 54(6), 431-436.

Martins, B. G., Silva, W. R. D., Maroco, J., \& Campos, J. A. D. B. (2019). Escala de Depressão, Ansiedade e Estresse: propriedades psicométricas e prevalência das afetividades. Jornal Brasileiro de Psiquiatria, 68, 32-41.

Melzack, R., \& Wall, P. D. (1965). Pain mechanisms: a new theory. Science, 150(3699), 971-979.

Meseguer, A. A., Fernández-de-las-Peñas, C., Navarro-Poza, J. L., Rodríguez-Blanco, C., \& Gandia, J. J. B. (2006). Immediate effects of the strain/counterstrain technique in local pain evoked by tender points in the upper trapezius muscle. Clinical Chiropractic, 9(3), 112-118.

Menezes, C. R. O., Moreira, A. C. P., \& Brandão, W. D. B. (2010). Base neurofisiológica para compreensão da dor crônica através da Acupuntura. Rev dor, 11(2), 161-8.

Misra, D. P., Agarwal, V., Gasparyan, A. Y., \& Zimba, O. (2020). Rheumatologists' perspective on coronavirus disease 19 (COVID-19) and potential therapeutic targets. Clinical rheumatology, 39(7), 2055-2062.

Mota, I. A., Oliveira, G. D. D., Morais, I. P. S., \& Dantas, T. F. (2021). Impact of COVID-19 on eating habits, physical activity and sleep in Brazilian healthcare professionals. Arquivos de Neuro-Psiquiatria.

National Institute For Health And Care Excellence - NICE. COVID-19 rapid guideline: managing the long-termeffects of COVID-19.

Noguchi, E. (2010). Acupuncture regulates gut motility and secretion via nerve reflexes. Autonomic neuroscience, 156(1-2), 15-18.

Nogueira, L. A. C. (2008). Neurofisiologia da terapia manual. Fisioterapia Brasil, 9(6), 414-421.

Pegoraro, A., Santos, M. E., Takamori, J. T., Carvalho, W. D. A. P. D., Oliveira, R. D., Barbosa, C. P., \& van Nimwegen, Â. (2019). Prevalência e intensidade da dor na histeroscopia diagnóstica em mulheres atendidas em uma clínica de infertilidade: análise de 489 casos. Einstein (São Paulo), 18.

Prestes, Y. A., dos Santos Souza, M., Pereira, M. S., Ervati, R., \& Campos, H. L. M. (2020). Confiabilidade intra e interexaminador da algometria para mensurar o limiar da dor em idosos institucionalizados. Revista Pesquisa em Fisioterapia, 10(3), 451-460.

Resende, L., Gomes, A., Tavares, A., Marques, J. P., Pinto, K., \& João, M. (2021). Bases neurofisiológicas da Acupuntura. Revista Portuguesa de Ciências Veterinárias, 116(617).

Rizzi Cintra, M. E., \& Figueiredo, R. (2010). Acupuncture and health promotion: possibilities in public health services. Interface-Comunicacao Saude Educacao, 14(32), 139-154

Salgado, A. S. I., \& Pereira, W. M. I. (2012). Influência aguda da mobilização do sistema nervoso autônomo na lombalgia. Conselho Científico, 10(49):277283.

Silva, J. R., Silva, M. L., \& Prado, W. A. (2011). Analgesia induced by 2 or $100 \mathrm{~Hz}$ electroacupuncture in the rat tail flick test depends on the activation of different descending pain inhibitory mechanisms. J. Pain, 12(1), 51-60.

Silva, R. M. V. D., \& Sousa, A. V. C. D. (2020). Fase crônica da COVID-19: desafios do fisioterapeuta diante das disfunções musculoesqueléticas. Fisioterapia em Movimento, 33.

Silveira, M. A. A., Martins, B. A., Chamon, L. S. F. G., Diniz, A. E. D., de Assis, J. B., Ferreira, L. D. T., ... \& de Castro Mendes, H. (2021). Aspectos das manifestações da síndrome pós-COVID-19: uma revisão narrativa. Revista Eletrônica Acervo Saúde, 13(12), e9286-e9286.

Takakura, N., Yajima, H., Takayama, M., Kawase, A., \& Homma, I. (2010). Inhibitory effect of needle penetration on vibration-induced finger flexion reflex in humans. Acupuncture in Medicine, 28(2), 78-82.

Takiguchi, R. S., Fukuhara, V. S., Sauer, J. F., Assumpção, A., \& Marques, A. P. (2008). Efeito da acupuntura na melhora da dor, sono e qualidade de vida em pacientes fibromiálgicos: estudo preliminar. Fisioterapia e Pesquisa, 15, 280-284.

Teixeira, C. N. G., de Queiroz Rodrigues, M. I., de Barros Silva, P. G., Barros, M. M. A. F., Alves, C. M. C., \& de Almeida, M. E. L. (2021). Qualidade de vida de estudantes de pós-graduação em Odontologia: uma análise por meio dos domínios do WHOQOL-bref. Revista da ABENO, $21(1), 1110$.

Teixeira, L., de Freitas, R. L., \& Carvalho, L. C. (2020). Os desafios do tratamento multidisciplinar da dor pós-pandemia ocasionada pelo vírus Sars-CoV-2. 
Research, Society and Development, v. 11, n. 2, e43811225969, 2022

(CC BY 4.0) | ISSN 2525-3409 | DOI: http://dx.doi.org/10.33448/rsd-v11i2.25969

Vas, J., Vega, E., \& Méndez, C. (2002). Tratamiento con acupuntura del dolor crónico benigno. Medicina de Familia (Andalucía), 4, 239-44.

Velavan, T. P., \& Meyer, C. G. (2020). The COVID-19 epidemic. Tropical medicine \& international health, $25(3), 278$.

White, A., Cummings, M., \& Filshie, J. (2018). An Introduction to Western Medical Acupuncture-E-Book. Elsevier.

Wolfe, F., Clauw, D. J., Fitzcharles, M. A., Goldenberg, D. L., Katz, R. S., Mease, P., \& Yunus, M. B. (2010). The American College of Rheumatology preliminary diagnostic criteria for fibromyalgia and measurement of symptom severity. Arthritis care \& research, 62(5), 600-610.

Ziani, M. M., Bueno, E. A., Kipper, L. R., Vendrusculo, F. M., Winck, A. D., \& Heinzmann-Filho, J. P. (2017). Efeitos da terapia manual sobre a dor em mulheres com fibromialgia: uma revisão de literatura. Ciência \& Saúde, 10(1), 48-55. 